\title{
Presencia de huevos de parásitos con potencial zoonótico en parques y plazas públicas de la ciudad de Temuco, Región de La Araucanía, Chile
}

\author{
Presence of parasite eggs with zoonotic potential in parks and public squares \\ of the city of Temuco, Araucanía Region, Chile \\ WA Armstrong ${ }^{\mathrm{a}^{*}}, \mathrm{C}$ Oberg $^{\mathrm{b}}, \mathrm{JJ}$ Orellana ${ }^{\mathrm{b}}$ \\ ${ }^{a}$ Escuela de Medicina Veterinaria, Universidad Santo Tomás, Temuco, Chile. \\ bFacultad de Medicina, Universidad de La Frontera, Temuco, Chile.
}

\begin{abstract}
SUMMARY
Many parasitic infections can be transmitted from dog to man, which constitutes a risk to public health worldwide. The aim of this study was to report any presence of parasite eggs in soil samples from 87 parks and public places in 6 areas of the city of Temuco, located between $37^{\circ} 24^{\prime}$ and $39^{\circ} 34^{\prime}$; latitude South, Region of Araucania, Chile. 193 three soil samples were collected, and 70 positive samples (36.3\%) were obtained by using the modified method of Fonrouge. In $48.3 \%$ of the parks, the eggs found corresponded to the genders Toxocara sp. (12.4\%), Taenia sp. (11.4\%) and eggs of Trichuris sp. (4.7\%). Also, a set of eggs identified as Strongylid (9.3\%) was found, but it was notpossible to determine whether they were parasites or free life helminthes. In addition, $2.8 \%$ of the samples showed polyparasitism. According to Fisher's exact test, there was no statistical association between the type of topsoil of the park and the type of contamination; and no differences were found between risk of infection and studied area. Results showed that parks and public places of Temuco are contaminated with parasite eggs mainly associated with dogs, posing a potential hazard to human health.
\end{abstract}

Palabras clave: parásitos, contaminación suelo, caninos.

Key words: parasites, soil pollution, dogs.

\section{INTRODUCCIÓN}

La expansión territorial ha creado nuevos asentamientos humanos, urbanizaciones y conjuntos habitacionales que traen consigo nuevas plazas o parques públicos a los que acuden adultos y niños para realizar actividades recreativas o de esparcimiento. Conjuntamente con el aumento de la población humana se produce un incremento en el número de mascotas (Velarde y col 1999).

Hay muchos agentes parasitarios transmisibles al ser humano y comportamientos como la geofagia, falta de higiene (Conde García y col 1989, Aguedelo y col 1990) y condiciones de saneamiento ambientales deficientes que posibilitan la exposición a la fuente infectiva (Gamboa y col 2009). Las áreas recreacionales como parques y plazas públicas son lugares donde las personas pueden tener contacto con elementos parasitarios causantes de zoonosis que provienen, principalmente, de las heces de perros vagos o perros (Vaughn y Jordan 1960, Prociv y Croese 1990, Croese 1995) cuyos propietarios tienen el hábito de pasear a su mascota para que defeque en estos lugares (Salinas y col 1987, Noemí y Rugiero 1998, Velarde y col 1999). Las viviendas, las calles, las plazas o cualquier área de alta concentración de personas y perros constituyen lugares

Aceptado: Aceptado: 15.12.2010.

* Manuel Rodríguez 060, Temuco, Chile; waldoarmstrong@ santotomas.cl donde las personas pueden tener contacto con heces que contienen elementos contaminantes (Glickman y Shantz 1981, Smith y col 1984, Zunino y col 2000, Larrieu y col 1997).

Desde el punto de vista de la salud pública los perros (con o sin propietarios) no sólo poseen importancia por sus mordidas, los accidentes de tránsito o la aversión que producen, sino que también debido a la contaminación ambiental de sus heces y/u orina y a los microorganismos patógenos que transportan en estos desechos orgánicos (Fok y col 2001).

Los estadios inmaduros de algunos parásitos del perro son eliminados en las heces, contaminando el suelo circundante. Para completar el ciclo los huevos deben ser ingeridos o incluso algunos pueden ingresar a través de la piel. En el hombre, que se comporta como hospedero accidental, se desarrollan distintas patologías dependiendo del agente etiológico, tales como: síndrome de larva migrante visceral, asociada principalmente a Toxocara canis (Acha 1986), pero autores como Noemí y Rugiero (1998), Milano y Oscherov (2002), Leguia (2002), TrilloAltamirano y col (2003) también lo asocian a Toxascaris leonina; síndrome de larva migrante cutánea, asociada principalmente a Ancylostoma spp. (Milano y Oscherov 2002) y en menor medida a Uncinaria spp. (Vanparijs y col 1991, Zúñiga y Llancapi 1993, Torno y col 1996, Urbina 1997) y otras patologías como infecciones intestinales (Milano y Oscherov 2002). 
La ciudad de Temuco es la capital de la Región de La Araucanía, región que presenta los más altos índices de ruralidad en el país, con un $38,7 \%$ en promedio, a lo que se suma una población mapuche importante, con características sociales y culturales propias ${ }^{1}$. Existen también altos índices de analfabetismo y pobreza, lo que favorece la permanencia de enfermedades zoonóticas como hidatidosis (Aliaga y Oberg 2000) y otras enfermedades como toxocariasis que se transmiten por falta de medidas higiénico-sanitarias, donde el contacto entre personas y restos de heces presentes en el suelo facilita el ingreso de elementos parasitarios causantes de éstas y otras enfermedades (Salinas y col 1987).

Un estudio realizado en la ciudad de Temuco por Oberg y col (2001), donde se recolectaron muestras fecales de perros en las calles, indica que en el $77 \%$ de ellas se detectó la presencia de parásitos del perro, de las cuales un 60\% era positiva a los géneros Toxocara sp. y Taenia sp., géneros a los que pertenecen especies de parásitos transmisibles al hombre. Los mismos autores señalan que aún después de desaparecidas las heces, quedan elementos parasitarios (huevos de parásitos) en el suelo, lo que constituye una fuente potencial de contaminación.

Herskovic y Astorga (1985) realizaron un estudio serológico en Chile sobre infecciones por Toxocara sp. en población humana adulta presuntamente sana, encontrando una seroprevalencia del 8,8\% distribuida en tres regiones: norte $\left(18-20^{\circ}\right.$ latitud Sur, $70-71^{\circ}$ longitud Oeste), centro (33-35 latitud Sur, $71^{\circ}$ longitud Oeste) y sur (53 latitud Sur, $74^{\circ}$ longitud Oeste), lo que sugiere una distribución importante dentro del país.

Se ha señalado que una prevalencia del $7 \%$ o más de T. canis en cualquier población de perros constituye un riesgo para la aparición de toxocariasis o "Larva migrante visceral" (Lamina 1974). Además, si consideramos que la toxocariasis canina tiene distribución en Latinoamérica con prevalencias que en México, Argentina, Brasil, Chile, Colombia y Perú varían entre 7 y 53\% (Alcaíno y Tagle 1970, Ehrhard y Kernbaum 1979), existe un riesgo latente de ser afectado por esta enfermedad (Minvielle y col 1993).

El estudio de la contaminación parasitaria del suelo es considerado un indicador directo del riesgo de contagio al que están expuestos los residentes de una región (Uga y col 1997), por lo que el objetivo del presente estudio fue detectar formas parasitarias, potencialmente patógenas para el ser humano, en muestras de suelo en parques y plazas públicas de la ciudad de Temuco, Región de La Araucanía, Chile.

http://www.censo2002.cl/, Instituto nacional de estadísticas, fecha consulta 06.11.2003.

\section{MATERIAL Y MÉTODOS}

La ciudad de Temuco está ubicada a 675 kilómetros al sur de Santiago, entre los $37^{\circ} 24^{\prime}$ y $39^{\circ} 34^{\prime}$ latitud Sur. El universo muestreal estaba conformado por $818.856 \mathrm{~m}^{2} \mathrm{de}$ áreas verdes distribuidas en 250 parques y plazas (información entregada por la Ilustre Municipalidad de Temuco). Este universo muestral representaba a todos los parques y plazas públicas de la ciudad, excluyendo aquellos con una superficie inferior a $500 \mathrm{~m}^{2}$.

En un parque o plaza no todas las áreas verdes están cubiertas de pasto, ya que existen sectores de equipamiento (bancos, piletas, basureros, otros), sectores con pérdida de la cubierta de pasto y sectores con presencia de ornamentos (árboles, arbustos y plantas). Por lo tanto, las áreas verdes con cobertura de pasto, que son los lugares donde las personas tienen una mayor probabilidad de entrar en contacto con heces o restos de heces, se denominaron áreas de riesgo.

Basándose en la observación en terreno de un parque o plaza pública estándar de la ciudad de Temuco, se determinó que las áreas de riesgo serían la cuarta parte de la superficie informada por la Municipalidad, es decir, de los $818.856 \mathrm{~m}^{2}$ de áreas verdes $204.714 \mathrm{~m}^{2}$ serían las áreas de riesgo.

Para sistematizar, enumerar y ordenar las áreas de riesgo a muestrear se definieron arbitrariamente zonas muestrales, que serían sectores dentro de las áreas de riesgo con una superficie de $4 \mathrm{~m}^{2}$, obteniendo un total de 12.795 zonas muestrales. Cada zona representaba la ubicación de un área de riesgo en una plaza o parque determinado. La metodología utilizada para el cálculo de zonas muestrales es inédita y se plantea como una alternativa para estudios parasitológicos del suelo.

El cálculo del tamaño de muestra se realizó según EPIINFO 6.0 asumiendo que la población de referencia está compuesta por 12.795 zonas muestrales, una prevalencia de contaminación de un $15 \%$ por datos observados en estudios como el de Fonrouge y col (2000) en suelos de la ciudad de La Plata, Argentina, y Oberg y col (2001) en heces diseminadas en la vía pública de la ciudad de Temuco, con una precisión en la estimación de la prevalencia de 5\% y un nivel de confianza para dicha estimación de un $95 \%$. El tamaño muestral calculado corresponde a 193 muestras.

La unidad elemental de muestreo o muestra corresponde al volumen de suelo que surge al recoger, con una pala, un cubo de $10 \mathrm{~cm}$ de largo, por $10 \mathrm{~cm}$ de ancho y $3 \mathrm{~cm}$ de profundidad, basándose en el método utilizado por Fonrouge y col (2000). Para tomar una muestra, cada zona muestral fue dividida en cuatro partes iguales (orientándose de norte a sur y de derecha a izquierda), de las cuales se tomó una al azar.

Las muestras se recolectaron entre los meses de agosto y noviembre del 2003 en bolsas de polietileno para su posterior procesamiento, almacenándose en un lugar fresco y seco por un período no mayor a diez días. 
DISTRIBUCIÓN Y SELECCIÓN DE LOS PARQUES Y PLAZAS A MUESTREAR

Temuco se divide en seis sectores o macrosectores (según la Ilustre Municipalidad de Temuco) que son: Centro (al que llamaremos sector 1), Amanecer (sector 2), Santa Rosa (sector 3), Pueblo Nuevo (sector 4), Poniente (sector 5) y Pedro de Valdivia (sector 6).

Las 193 muestras se obtuvieron de 87 parques y plazas de la ciudad de Temuco. En el cuadro 1 se muestra el número total de parques y plazas por sector y las muestras que se tomaron. La cantidad de muestras fue proporcional a la superficie de áreas verdes, no al número de parques o plazas existentes por sector, ya que algunos sectores tenían una menor cantidad de parques pero con una superficie mayor.

\section{PROCESAMIENTO DE LAS MUESTRAS}

Cada muestra se homogeneizó en la misma bolsa en la que se obtuvo, agregándole un volumen de $60 \mathrm{ml}$ de agua. De ésta, se extrajeron cuatro alícuotas de $5 \mathrm{~g}$ que se diluyeron individualmente en $50 \mathrm{ml}$ de agua; se filtraron por un colador de alambre, previamente flameado, recubierto de un trozo de gasa y el filtrado se recolectó en un vaso de $250 \mathrm{ml}$. Posteriormente, se sedimentó por 20 minutos y el sedimento se recogió en un tubo de centrífuga, el cual nuevamente se dejó sedimentar por 5 minutos, se eliminó el sobrenadante y al sedimento se le agregó una solución de sulfato de zinc sobresaturado $(\mathrm{d}=1,20)$, que se centrifugó a 2000 r.p.m. durante 5 minutos.

Posterior al centrifugado, cada tubo se llenó con la solución de sulfato de zinc hasta formar un menisco y se dejó flotar por 10 minutos con un cubreobjeto, para finalmente observar al microscopio en aumento de $10 \mathrm{x}$.

Finalmente, una muestra sería informada como positiva independiente de la cantidad o del tipo de huevos que se encontrara en ella.

\section{ANÁLISIS ESTADÍSTICO}

Para el análisis de los datos se utilizaron los siguientes estadísticos:

- Intervalo de confianza 95\%.

- Odds Ratio: Estimación de riesgo mediante una regresión logística. En este caso se usó para determinar las diferencias de riesgo entre los lugares a estudiar. Un valor de 0 indica que existe menor riesgo de un lugar en comparación con otro, un valor de 1 indica que no hay diferencias de riesgo entre los lugares comparados y un valor mayor a 1 indica que este "lugar" es tantas veces más riesgoso que el otro.

- P>|z|: Indica en porcentaje la exactitud de la medición, en este caso se realizó con un valor de $\mathrm{z}=0,05$.

Además, para determinar diferencias estadísticas significativas entre los valores obtenidos en las mediciones se recurrió la prueba exacta de Fisher (Fisher's exact test). Todos estos datos fueron analizados por el programa estadístico STATA® v7.0.

\section{RESULTADOS}

De los 87 parques y plazas públicas muestreados, 42 resultaron positivos a huevos de helmintos $(48,3 \%)$. El cuadro 1 muestra en detalle el número y porcentaje de parques y plazas públicas que resultaron positivas para huevos de helmintos. La distribución de las muestras positivas en los distintos sectores de la ciudad de Temuco se indican en la figura 1.

Los huevos de helmintos detectados corresponden a los géneros Toxocara sp., Taenia sp., Trichuris sp. y un conjunto de huevos tipo estrongílido que no pudieron ser diferenciados en forma visual. Toxocara sp. es el helminto que se encuentra con mayor frecuencia $(12,4 \%) \mathrm{y}$ Trichuris sp. con menor frecuencia $(4,7 \%)$ (cuadro 2 ).

El cuadro 1 muestra en número $\left(\mathrm{N}^{\circ}\right)$ y porcentaje $(\%)$ las muestras positivas a huevos de helmintos $(\mathrm{n}=$ total de

Cuadro 1. Número y porcentaje de parques y plazas públicas positivos para helmintos entre 87 muestreados en la ciudad de Temuco, Región de La Araucanía, Chile.

Number and percentage of parks and public squares positive for helminthes among 87 samples in the city of Temuco, Araucanía Region, Chile.

\begin{tabular}{|c|c|c|c|c|}
\hline Sector & $\mathrm{N}^{\mathrm{o}}$ plazas & Plazas positivas (\%) & $\begin{array}{l}\text { Muestras tomadas } \\
\text { por sector }\end{array}$ & Muestras positivas (\%) \\
\hline 1 & 10 & $6(60)$ & 43 & $17(39,5)$ \\
\hline 2 & 13 & $6(46,2)$ & 30 & $12(40)$ \\
\hline 3 & 5 & $4(80)$ & 27 & $11(40,7)$ \\
\hline 4 & 8 & $4(50)$ & 15 & $4(26,7)$ \\
\hline 5 & 38 & $16(42,1)$ & 58 & $18(31)$ \\
\hline 6 & 13 & $6(46,2)$ & 20 & $8(40)$ \\
\hline Total & 87 & $42(48,3)$ & 193 & $70(36,3)$ \\
\hline
\end{tabular}




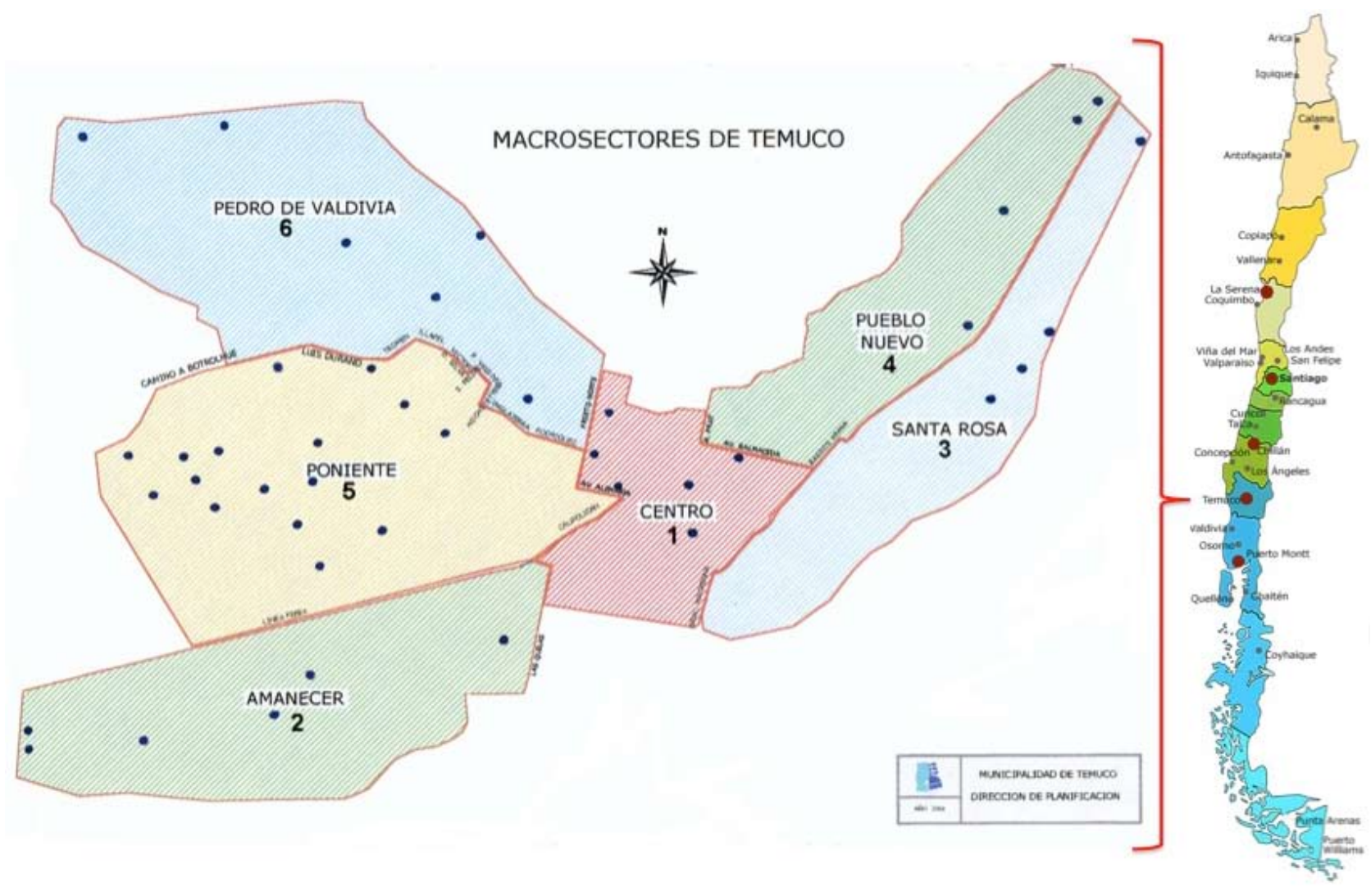

Figura 1. Distribución de los parques o plazas positivos a huevos de helmintos, de un total de 87 parques o plazas públicas muestreados en la ciudad de Temuco, Región de La Araucanía Chile.

Distribution of parks and squares positive for helminthes eggs, from a total of 87 parks and public squares sampled in the city of Temuco, Araucanía Region, Chile.

Cuadro 2. Porcentaje de huevos de helmintos en parques y plazas públicas de la ciudad de Temuco, Región de La Araucania, Chile $(\mathrm{n}=193)$.

Percentage of helminthes eggs in public parks of the city of Temuco, Araucania Region, Chile ( $\mathrm{n}=193)$.

\begin{tabular}{cc}
\hline Helminto & \% huevos encontrados \\
\hline Toxocara sp. & 12,4 \\
Taenia sp. & 11,4 \\
Estrongílidos & 9,3 \\
Trichuris sp. & 4,7 \\
Total & 37,8 \\
\hline
\end{tabular}

muestras tomadas por sector) encontrados en las muestras de suelo de parques y plazas públicas de Temuco.

De las 70 muestras positivas $(n=193)$, en 2 de ellas se encontró poliparasitismo, observándose las siguientes asociaciones: en una de ellas se encontraron huevos de los géneros Toxocara sp., Taenia sp. y tipo estrongílido y en la segunda se encontró la presencia de huevos del género Taenia sp. y tipo estrongílido.

El cuadro 3 muestra la comparación de riesgo entre los sectores, no encontrándose diferencias significativas
Cuadro 3. Riesgo estimativo (Odds Ratio) entre la superficie y la presencia de huevos de helmintos en los seis sectores de la ciudad de Temuco, Región de La Araucanía, Chile.

Risk estimate (Odd Ratio) between the surface and the presence of helminthes eggs in the six sectors of the city of Temuco, Araucanía Region, Chile.

\begin{tabular}{ccc}
\hline Sectores de Temuco & Odds Ratio & $\mathrm{P}>|0,05|$ \\
\hline 1 & 0,99 & 0,97 \\
2 & 1,02 & 0,98 \\
3 & 1,06 & 0,92 \\
4 & 0,55 & 0,42 \\
5 & 0,68 & 0,48 \\
\hline
\end{tabular}

entre ellos. Para realizar este análisis, todos los sectores se compararon con el sector 6 escogido aleatoriamente por STATA 7.0.

\section{DISCUSIÓN}

Debido a que no existen antecedentes anteriores sobre contaminación del suelo en Temuco, el único estudio que entrega antecedentes de interés es el de Oberg y col (2001), donde se evidenció que el $77 \%$ de las muestras 
de heces diseminadas en la vía pública de Temuco estaban positivas a huevos de helmintos parásitos. Si bien la metodología utilizada en dicho estudio se basa en determinación de parásitos en heces, Gamboa y col (2009) indican que geohelmintos como T. Canis puede permanecer viable en el suelo varios años, por lo que aun después de que las heces desaparecieran del suelo se esperaba encontrar huevos de parásitos en las muestras de suelo analizadas.

De los 42 parques y plazas que resultaron positivos a huevos de helmintos $(n=87)$, el sector Poniente, en donde se encuentra la mayor cantidad de parques y plazas (38 parques), es también el que registra la mayor cantidad de parques contaminados $(38,1 \%)$. Sin embargo, al realizar la comparación de riesgo, no se aprecian diferencias significativas entre la contaminación de los parques y plazas de este sector y los otros sectores. Estudios como el de Gamboa y col (2009) sugieren una fuerte asociación entre parasitosis y condiciones socioeconómicas, relacionado principalmente con el no retiro de las heces de las mascotas en espacios públicos (Conde García y col 1989, Aguedelo y col 1990). Según los datos entregados por la Municipalidad de Temuco, el sector Poniente es el que agrupa a familias con mayores ingresos y los sectores Santa Rosa y Amanecer agrupan a las familias de menores ingresos de la ciudad. Sin embargo, el riesgo es similar en los seis sectores (cuadro 3) por lo que factores como la tenencia responsable de mascotas, cuidados sanitarios, costumbres sanitarias como el retiro de heces de la vía pública y vagancia de caninos no tendrían mayores variaciones entre los distintos sectores de la ciudad, a pesar de las diferencias socioeconómicas entre ellos.

De las 193 muestras recolectadas, un $28,5 \%$ de éstas contenían algún huevo identificado dentro de los géneros Toxocara sp., Taenia sp. y Trichuris sp., de los cuales los dos primeros presentan especies causantes de zoonosis parasitarias, como Toxocara canis y Echinococcus granulosus (Oberg y col 1979).

Si bien los géneros Toxocara sp., Taenia sp. y otros asociados con huevos tipo estrongílido están presentes en las heces de los gatos, éstos prefieren defecar en suelos arenosos donde puedan enterrar sus deyecciones (Uga y col 1996), por lo tanto, los resultados obtenidos sugieren que el perro doméstico sería la fuente principal de contaminación parasitaria del suelo de parques y plazas públicas. Esto se corrobora con los resultados obtenidos por López y col (2005) en Perú y Cazorla y col (2007) en Venezuela, donde asocian al perro como el principal agente contaminante de parques y plazas. Además, se complementa con la observación de campo realizada al momento de tomar las muestras, donde se detectaron perros y restos de sus heces en diferentes estados de descomposición diseminadas en los distintos parques y plazas públicas muestreados.

La técnica utilizada para el procesamiento de las muestras se basó en la descrita por Fonrouge y col (2000). Sin embargo, debido a que esta técnica no había sido validada en el laboratorio se realizaron pruebas preliminares con muestras de suelo contaminadas intencionalmente con huevos de T. canis para ver la capacidad de recuperación de huevos, pero luego de procesar varias muestras solo se recuperaron uno o dos huevos en muestras de tierra con una alta carga de huevos. Con el objeto de aumentar la cantidad de muestra procesada y por ende la probabilidad de detectar los huevos, se utilizaron 4 alícuotas de $5 \mathrm{~g}$ en vez de una de $10 \mathrm{~g}$ y se cambió la solución de azúcar por sulfato de zinc para hacer la flotación, tal como lo utilizó Salinas y col (2001) en su estudio de contaminación de plazas en la ciudad de Santiago. A esto se sumó el menor costo económico asociado a los insumos y materiales al comparar este procedimiento con otras técnicas descritas para la recuperación de huevos en muestras de suelo (Fonrouge y col 2000, Ruiz de Ybáñez y col 2000, Córdoba y col 2002).

$\mathrm{Al}$ analizar otros aspectos de la técnica utilizada para el procesamiento de las muestras, la densidad de la solución de sulfato de zinc utilizada para realizar la flotación y recuperación de huevos es levemente más baja que la recomendada por Ruiz de Ybáñez y col (2000), quienes sugieren una densidad de 1,27 para realizar las flotaciones recuperando una mayor cantidad de huevos de T. canis. En este estudio se utilizó una densidad de 1,20, lo que podría haber influido en la menor flotación de huevos y por ende en su menor recuperación. Ruiz de Ybáñez y col (2000) también observaron que el lavado ideal de la unidad de muestra debía realizarse con agua destilada, ya que el utilizar agua potable como se hizo en el presente estudio, podría disminuir la recuperación de huevos de helmintos.

Los principales factores que influyen en la recuperación de huevos son: a) el método utilizado, que en este caso y como explicaron los autores entregaba buenos resultados, especialmente en la recuperación de huevos de $T$. canis $\mathrm{y}$ a un costo menor de insumos y materiales; $b$ ) factores ambientales como temperatura y humedad relativa, factores que determinan una mayor o menor viabilidad de los huevos de helmintos con una etapa de vida libre o necesitan del medio ambiente para madurar a un estado infectante (Dada y Lindquist 1979, Ruiz de Ybáñez y col 2000) y c) la textura del suelo, ya que en el suelo arenoso es donde se registra la mayor recuperación de huevos de T. canis (Nunes y col 1994). En relación a este último punto la ciudad de Temuco presenta principalmente suelos arcillosos, que son menos porosos que los arenosos (Honorato 2000). Este factor podría haber generado una menor recuperación de huevos, a pesar de que Cazorla y col (2007) no encontraron relación estadística entre el tipo de suelo y recuperación de huevos.

En relación a la temperatura y humedad presentes al momento de recolectar las muestras, las temperaturas promedio registradas en la ciudad de Temuco entre agosto y noviembre se sitúan entre los 8 y $14{ }^{\circ} \mathrm{C}$, con una humedad relativa de entre un 83 y $86 \%$ (Laboratorio 
Meteorológico de la Universidad Católica de Temuco), condiciones favorables para los huevos de parásitos, ya que por ejemplo, para el caso de Toxocara sp., temperaturas de entre 8 y $35^{\circ} \mathrm{C}$ (Boch 1982) y una humedad entre 85 y $95 \%$ (Soulsby 1965) son favorables para su desarrollo (López y col 2005, Cazorla y col 2007).

Si bien no se encontraron huevos larvados y tampoco se realizó una determinación de viabilidad de los mismos, estudios como el de Minvielle y col (1993) señalan que la presencia de elementos parasitarios del perro en parques y plazas públicas es un indicador de riesgo para niños y adultos. Además, se ha demostrado que los huevos de T. canis pueden infectar a un hospedero susceptible después de estar en el suelo un largo tiempo en condiciones extremas de temperatura y humedad (Gamboa y col 2009). Los resultados obtenidos en estudios similares en Latinoamérica son muy variables. En Chile se han encontrado prevalencias entre 4,5 a 51,9\%, en Basil entre 0,7 a 23,6\%, Argentina de 5 a 18\% y México de 0,7 a 37,3\% (Trillo-Altamirano y col 2003). En vista de los antecedentes existentes se puede suponer que una parte importante de los huevos encontrados en el presente estudio, especialmente del género Toxocara sp., si bien no estaban larvados, pueden haber sido viables y por tanto potenciales causantes de zoonosis en la población.

En Lima (Perú), Velarde y col (1999) encontraron un $37 \%$ de positividad a huevos de Toxocara sp.; en Sao Paulo, Brasil, Santarem y col (1998) encontraron un 17,3\% para el mismo parásito. En otros estudios en ciudades de Europa y América entre 1973 y 1984 varían sus frecuencias de hallazgo de huevos para Toxocara sp. en muestras de tierra entre 8,7 y 60\% (Salinas y col 1987). Cazorla y col (2007) encontraron un $60 \%$ de muestras positivas a huevos de Toxocara sp. en parques de Venezuela y resultados similares en la recuperación de los huevos de Toxocara sp. obtuvieron Castillo y col (2001), y Loh y Israf (1998) en estudios de suelos realizados en Perú y Malasia, respectivamente. Por otra parte, en un estudio realizado en la ciudad de La Plata, Argentina (Córdoba y col 2002) donde utilizaron una unidad elemental de muestra de $400 \mathrm{~cm}^{2}$ por $2 \mathrm{~cm}$ de profundidad, muy superior a la utilizada en este estudio y a la utilizada por Fonrouge y col (2000), encontraron una positividad de un 56,5\% para Toxocara sp., 21\% para Trichuris sp. y $13 \%$ para Uncinaria sp. y Dipylidium caninum. Estos hallazgos, si bien son mayores a los encontrados en este trabajo para Toxocara sp. y Trichuris sp., poseen diferencias en cuanto a la unidad elemental de muestra utilizada y al total de muestras recolectadas $(n=140)$. Además, en el presente estudio no se encontraron huevos de $D$. caninum ni se hizo una diferenciación de los huevos tipo estrongílido para determinar géneros como Uncinaria sp. Queda la interrogante del método diagnóstico utilizado en el trabajo de Córdoba y col (2002) para realizar dicha diferenciación, ya que en la literatura no hay claves para la diferenciación visual entre huevos de estrongílido parásitos o de vida libre en muestras de suelo, por lo que este $13 \%$ que reportan como Uncinaria sp. sería similar al 9,33\% encontrado en el presente estudio.
En relación a otros resultados, como ya se mencionó, se obtuvo un $11,4 \%$ de positivos para huevos de Taenia sp., superior al 5,9\% informado por Oberg y col (2001) y 2 puntos más bajos que Córdoba y col (2002) que encontraron un $13 \%$ en la ciudad de La Plata, Argentina. Llama la atención que el resultado fuera superior al de Oberg y col (2001), ya que al pasar los huevos de las heces al suelo se espera una mayor dispersión de los elementos parasitarios cuando se diseminan las heces. Si bien Sánchez y col (2003) indican una supervivencia de huevos de Taenia spp. por períodos de 300 días a temperaturas entre $0{ }^{\circ} \mathrm{C}-10{ }^{\circ} \mathrm{C}$ con $85 \%$ de humedad relativa, se esperaba encontrar una menor cantidad de muestras positivas de Taenia.

Aunque no se pudo establecer la(s) especie(s) de Taenia sp. a la que pertenecía este $11,4 \%$ encontrado en el presente estudio, hay que señalar que en Chile el perro porta varias especies de taenias, tales como T. hydatigena, T. serialis, T. multiceps, T. pisiformis y Echinococcus granulosus (Alcaíno y Gorma 1999). Esta última es causante de la hidatidosis, que es una zoonosis parasitaria producida por el estado larval de E. granulosus, Cestodo de alta prevalencia en perros urbanos y rurales de Chile, especialmente aquellos que habitan en regiones con una gran actividad agrícola como la Región de La Araucanía. En esta región la tasa humana es de 45 x 100.000 habitantes (Aliaga y Oberg 2000). Debido a que la ciudad de Temuco tiene un estrecho contacto con zonas rurales, existe la posibilidad de que algunos de los huevos identificados como Taenia sp. podrían corresponder a E. granulosus. La detección de estos elementos infectantes, independientemente de la taxonomía de dichos huevos, puede representar un riesgo para la salud de la población humana (Sánchez y col 2003).

En lo referente a Trichuris sp., si bien no tiene importancia directa en el área de salud pública debido a que presentan especificidad en cuanto a hospedero, su hallazgo es de cuidado debido a que son indicadores de contaminación ambiental por heces (Oberg y col 2001) y aunque fue el género que se encontró en menor cantidad, hay que precisar que son huevos resistentes y de larga vida (Boch y Supperer 1982), por lo que su hallazgo puede indicar una contaminación previa a la de este estudio reflejando una permanencia de las heces en parques y plazas hasta su descomposición y asimilación por el suelo.

Los huevos tipo estrongílido encontrados en este estudio no pudieron ser diferenciados en géneros, ni menos en especies, debido a que no es posible diferenciarlos visualmente, pero algunos de ellos podrían corresponder a la Familia Ancylostomidae, a la que pertenece el género Uncinaria sp., ya que Oberg y col (2001) señalan una prevalencia para Uncinaria sp. de 59,5\% en muestras de heces en la ciudad de Temuco. Con estos antecedentes se podría esperar que un porcentaje o la totalidad del 9,33\% de huevos tipo estrongílido encontrados, correspondería a Uncinaria sp. Sin embargo, no se puede descartar que éstos pertenezcan a estrongílidos de vida libre (Rodríguez y col 2009) o incluso a especies no descritas de nematodos 
(Asaolu y col 1992, Córdoba y col 2002), ya que de las diferentes formas de vida presentes en el suelo, entre ellas nematodos de vida libre, se estima que gran parte de ellas permanecen sin identificar (Alexander 1977).

El síndrome de larva migrante cutánea ha sido descrita en la población de Chile, pero es poco frecuente debido a que esta zoonosis es producida principalmente por Ancylostoma braziliensis y Ancylostoma caninum y muy raramente por Uncinaria stenocephala (Schenone 1987, Alcaíno y Gorman 1999), pero debido a que este parásito se asocia a una zoonosis, especialmente en niños que acostumbran a jugar con perros y en lugares públicos y en adultos que manipulan tierra (Alcaíno y Gorman 1999), no se debe descartar el riesgo potencial de transmisión.

Los resultados permiten concluir que los parques y plazas públicas de la ciudad de Temuco están contaminados con huevos de parásitos asociados principalmente a perros, lo que es potencialmente riesgoso para la salud de las personas debido a que algunos de los géneros identificados incluyen a especies zoonóticas.

\section{RESUMEN}

Muchas infecciones parasitarias del perro pueden ser transmitidas al hombre, lo cual constituye un riesgo para la salud pública a nivel mundial. El objetivo de este estudio fue detectar la presencia de huevos de parásitos en muestras del suelo en 87 parques y plazas públicas distribuidas en seis sectores de la ciudad de Temuco localizada entre los $37^{\circ} 24^{\prime}$ y 39³4' lat. Sur, Región de La Araucanía, Chile. Se tomaron 193 muestras de suelo, obteniendo 70 muestras positivas $(36,3 \%)$ mediante el método Fonrouge modificado. En el 48,3\% de los parques se encontraron huevos de los géneros Toxocara sp. $(12,4 \%)$, Taenia sp. $(11,4 \%)$ y huevos de Trichuris sp. (4,7\%). También se identificó un conjunto de huevos tipo estrongílido correspondiente a un 9,3\%, no pudiendo determinar si se trataba de helmintos parásitos o de vida libre. Además, el 2,8\% de las muestras presentó poliparasitismo. Según el test exacto de Fisher, no hubo asociación estadística entre el tipo de superficie de los parques y la contaminación presente, tampoco se detectaron diferencias para riesgo de contagio entre los sectores estudiados. Los resultados permiten concluir que los parques y plazas públicas de Temuco están contaminados con huevos de parásitos asociados principalmente a perros, lo que es potencialmente riesgoso para la salud humana.

\section{REFERENCIAS}

Acha P. 1986. Zoonosis y enfermedades transmisibles comunes al hombre y otros animales. $2^{\text {da }}$ ed. Publicación Científica OPS, Washington D.C., EEUU.

Aguedelo C, E Villarreal, E Cáceres, C López, J Eljach, N Ramírez, C Hernández, A Corredor. 1990. Human and dogs Toxocara canis infection in a pool neighborhood in Bogota. Mem I Oswaldo Cruz $85,75-78$

Alcaíno H, I Tagle. 1970. Estudio de enteroparasitosis del perro en Santiago. Bol Chil Parasitol 25, 5-8.

Alcaíno H, T Gorman. 1999. Parásitos de los Animales Domésticos en Chile. Parasitol día 23, 33-41.

Alexander M. 1977. Introduction to soil microbiology. John Wiley \& Sons, New York, USA, Pp 472-499.

Aliaga F, C Oberg. 2000. Epidemiología de la hidatidosis humana en la IX región de La Araucanía, Chile, 1991-1998. Bol Chil Parasitol 55, 54-58.
Asaolu SO, CV Holland, JO Jegede, NR Fraser, RC Stoddard, DW Crompton. 1992. The prevalence and intensity of soil-transmited helminthiases in rural communities in southern Nigeria. Ann Trop Med Parasitol 86, 279-287.

Boch J, R Supperer. 1982. Parasitología en Medicina Veterinaria. Hemisferio Sur, Buenos Aires, Argentina.

Castillo D, C Paredes, C Zañartu, G Castillo, R Mercado, V Muñoz, H Schenone. 2000. Contaminación ambiental por huevos de Toxocara sp. en algunas plazas y parques de la ciudad de Santiago, Chile, 1999. Bol Chil Parasitol 55, 3-4.

Castillo Y, H Bazán, D Alvarado, G Sáez. 2001. Estudio epidemiológico de Toxocara canis en parques recreacionales del distrito de San Juan de Lurigancho, Lima-Perú. Parasitol día 25, 109-114.

Cazorla D, P Morales, M Acosta. 2007. Contaminación de suelos con huevos de Toxocara spp. (nematoda, ascaridida) en parques públicos de la ciudad de Coro, Estado Falcón, Venezuela. Revista Científica FCV-LUZ 17, 117-122.

Conde García L, A Muro-Alvarez, FS Martín. 1989. Epidemiological studies on toxocariasis and visceral larva migrans in a zone of Western Spain. Am Trop Med Parasitol 83, 621.

Córdoba A, M Ciarmela, B Pezzani, M Gamboa, M De Luca, M Minvielle, JA Basualdo. 2002. Presencia de parásitos intestinales en paseos públicos urbanos en La Plata Argentina. Parasitol latinoam 57, 25-29.

Croese J. 1995. Seasonal influence o human enteric infection by Ancylostoma caninum. Am J Trop Med Hyg 53, 158-161.

Dada BJ, WD Lindquist. 1979. Studies on flotation techniques for the recovery of helminth eggs from soil and the prevalence of eggs of Toxocara spp in some Kansas public places. J Am Vet Med Assoc 174, 1208-1210.

Ehrhard, T, S Kernbaum. 1979. Toxocara canis et toxocarose humaine. Bull Inst Pasteur 77, 225-287.

Fok E, V Szatmari, K Busak, F Rozgonyi. 2001. Prevalence of intestinal parasites in dogs in urban and rural areas of Hungary. Vet Quart 23, 96-98.

Fonrouge R, M Guardis, N Radman, S Archelli. 2000. Contaminación de suelos con huevos de Toxocara sp. en plazas y parques públicos de la ciudad de La Plata. Buenos Aires, Argentina. Bol Chile Parasitol 55, 83-85.

Gamboa MI, LE Kozubsky, ME Costas, M Garraza, MI Cardozo, ML Susevich. 2009. Asociación entre geohelmintos y condiciones socioambientales en diferentes poblaciones humanas de Argentina. Rev Panam Salud Pública 26, 1-8.

Glickman T, PM Shantz. 1981. Epidemiology and pathogenesis of zoonotic toxocariasis. Epidem Rev 3, 230.

Herskovic P, B Astorga. 1985. Toxocariasis humana en Chile. Rev Med Chile 113, 18-21.

Honorato R. 2000. Manual de edafología. $4^{\text {a }}$ ed. Universidad Católica de Chile Ediciones, Santiago, Chile.

Lamina J. 1974. Immunodiagnosis of visceral larva migrans in man. In: Soulsby EJ (ed). Parasitic zoonoses, clinical and experimental studies. Academic Press INC, London, UK.

Larrieu E, J Alvarez, E Cavagion, J Lamberti, C Calvo, A Herrasti, M Cachau, L Gino. 1997. Estudio descriptivo de la contaminación por materia fecal de pequeños animales en áreas urbanas de General Pico, Argentina. Vet Arg 14, 198.

Leguia PG. 2002. Enfermedades parasitarias de perros y gatos. Epidemiología y control. $2^{\mathrm{a}}$ ed. Editorial de Mar EIRL, Lima, Perú, Pp 150-155.

Loh A, D Israf. 1998. Tests on the centrifugal flotation technique and its use in estimating the prevalence of Toxocara in soil samples from urban and suburban areas of Malaysia. J Helminthol 72, 39-42.

López F, A Chávez, E Casas. 2005. Contaminación de los parques públicos de los distritos de Lima oeste con huevos de Toxocara sp. Rev Inv Vet 16, 76-81. 
Milano A, EB Oscherov. 2002. Contaminación por parásitos caninos de importancia zoonótica en playas de la ciudad de Corrientes, Argentina. Parasitol latinoam 57, 119-123.

Minvielle MC, BC Pezzani, JA Basualdo. 1993. Frecuencia de hallazgo de huevos de helmintos en materia fecal canina recolectada en lugares públicos de la ciudad de La Plata, Argentina. Bol Chil Parasitol 48, 63-65.

Noemí I, E Rugiero. 1998. Larvas migrantes. En: Atías A (ed). Parasitología Médica $3^{\mathrm{a}}$ ed. Publicaciones Técnicas Mediterráneo Ltda. Santiago, Chile, Pp 332-337.

Nunes C, I Sinhorini, S Ogassawara.1994. Influence of soil texture in the recovery of Toxocara canis eggs by a flotation method. Vet Parasitol 53, 269-274.

Oberg, C, R Franjola, V Leyan. 1979. Helmintos del perro doméstico (Canis familiaris) en la ciudad de Valdivia, Chile. Bol Chil Parasitol 34, 21-26.

Oberg C, C Herrera, J Moreno, F Fonseca. 2001. Parásitos del perro, problema ambiental y salud pública. I Congreso Chileno de Bioanálisis

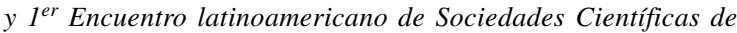
Laboratorio y Banco de sangre, Iquique, Chile.

Prociv P, J Croese. 1990. Human eosinophilic enteritis caused by dogs hookwoorm Ancylostoma caninum. Lancet 335, 1299-1302.

Rodríguez JG, JL Olivares, J Arece, E Roque. 2009. Evolución de los parásitos: Consideraciones generales. Rev Salud Anim 31, 13-17.

Ruiz de Ybáñez MR, M Garijo, M Goyena, FD Alonso. 2000. Improved methods for recovering eggs of Toxocara canis from soil. J Helminthol 74, 349-353.

Salinas P, L Reyes, MT Sotomayor, T Lentoja. 1987. Prevalencia de huevos de Toxocara sp. en algunas plazas y parques públicos de la Región Metropolitana de Santiago de Chile. Bol Chil Parasitol 42, 33-36.

Salinas P, H Matamala, H Schenone. 2001. Prevalencia de hallazgo de huevo de Toxocara canis en plazas de la Región Metropolitana de la ciudad de Santiago Chile. Bol Chil Parasitol 56, 3-4.

Sánchez P, S Raso, C Torrecillas, I Mellado, A Ñancufil, CM Oyarzo, ME Flores, M Córdoba, MC Minvielle, JA Basualdo. 2003. Contaminación biológica con heces caninas y parásitos intestinales en espacios públicos urbanos en dos ciudades de la Provincia de Chubut. Patagonia Argentina. Parasitol Latinoam 58, 131-135.
Santarem V, I Sartor, F Bergamo.1998. Contaminação, por ovos de Toxocara spp, de parques e praças públicas de Botucatu, São Paulo, Brasil. Rev Soc Brasil Med Trop 31, 529-532.

Schenone H. 1987. Parasitosis humanas que pueden ser causadas o transmitidas por mascotas domésticas en Chile. Bol Chil Parasitol 42, 16-23.

Smith R, H Hagstad, GB Berd. 1984. Visceral larva migrans: risk assessment in Baton Rouge, Louisiana. Int J Zoonoses 11, 189.

Soulsby E. 1965. Textbook of Veterinary Clinical Parasitology. Vol 1. Blackwell Scientific Publications, Oxford, England, Pp 28-30.

Torno O, S García, M Prat, B Santamaría. 1996. Enteroparásitos del perro en un sector de Bahía Blanca, Argentina. Parasitol al Día 20, 144-146.

Trillo-Altamirano M, A Carrasco, F Cabrera. 2003. Prevalencia de helmintos enteroparásitos zoonóticos y factores asociados en Canis familiaris en una zona urbana de la ciudad de Ica, Perú. Parasitol Latinoam 58, 136-141.

Uga S, T Minami, K Nagata. 1996. Defecation habits of cats and dogs and contamination by Toxocara eggs in public park sandpits. Am J Trop Med Hyg 54, 122-126.

Uga S, W Nagnae, V Chongsuvivatwong. 1997. Contamination of soil with parasite eggs and oocysts in Southern Thailand. Southeast Asian J trop Med Public Health 24, 14-17.

Urbina F. 1997. Larva Migrans Cutánea. Bol Hosp San Juan de Dios 44, 313-317.

Vanparijs O, L Hermans, L Van Der Flaes.1991. The level of helminth and protozoal infection in strays and web-caved-for dogs and cats in Belgium from 1980 to 1990 was investigated. Vet Parasitol 38, 67-73.

Vaughn J, R Jordan. 1960. Intestinal nematodes in well-cared-for dogs. Arm J trop Med 9, 29.

Velarde J, A Chávez, E Casas. 1999. Contaminación de parques públicos de la provincia constitucional del Callao con huevos de Toxocara spp. Rev Inv Vet Peru 10, 12-15.

Zunino M, M de Francesco, A Kuruc, N Schweigmann, M WisniveskyColli, O Jenesn. 2000. Contaminación por helmintos en espacios públicos de la Provincia de Chubut, Argentina. Bol Chil Parasitol $55,78-83$.

Zúñiga J, P Llancapi. 1993. Larva Migrans Cutánea. Dermatología 9, 110 . 